\title{
Musings on Event Timing for the ATR
}

This note summarizes discussions that I have had over the last two weeks on requirements for the timing system. This is not intended to be a final solution, but rather a record of the discussions tinged by my interpretation.

Unlike the mostly asynchronous requirements for the RHIC rings, the ATR functions must be synchronized with AGS events. For example, beam should only be extracted during a short AGS flattop. Fig. 1 shows hypothetical time-lines of the AGS and switching magnet ramps for two AGS cycles in addition to three kicker pulses for three extracted bunches per cycle. The vertical dashed lines indicate the potential timing events: T0 for the start of the AGS cycle, Tf for the beginning of the flattop, Td for the end of the flattop, Te for the end of the AGS cycle, and Tk for the kicker warning events.

The most important event Tk is one which warns that the kicker is about to fire, so that the electronics cards for BPM's, etc., can be armed to take data. There might be anywhere from 0 to 12 of these in a given AGS cycle.

Another important event would be one to identify the beginning of an AGS cycle for extraction into the U-line. This could be derived from either the AGS TO event or the prepulse event.

The end of the AGS cycle should be indicated by another type of event (Te). This event could be used to signal the frame grabbers to send the data to the top level application codes. Just counting the expected number of events is not necessarily enough. If, for example, three pulses were requested for this cycle, but the kicker charging system aborted on one, then we might be left waiting for an event which will never come.

In order to start the down ramp of the switching magnet, we should have an event generated by the down ramp of the AGS (Td). This event could also serve the purpose of the end of cycle event, since no extraction should be done after the down ramp begins.

Reversing of the switching magnet's polarity takes place when the field is ramped down and a zero current is sensed, so this does not require a special event. A bit can be set in the power supply control to disable the polarity reversal. The magnet can ramp in about 0.5 seconds, and the reverse switch should occur within 0.2 seconds. These times are in line with the AGS ramp times and the usual minimum time between the end of one AGS cycle and start of the next.

An event (Tf) for the beginning of the flattop could be defined, but I do not see a compelling reason for this at present.

Jörg Kewisch requested that several events be reserved for the WFG's. This will have to be thought about in more detail. Quite possibly more than one type of event may be needed, but we should be careful not to define too many without knowing how they will be used.

Tom Shea wants to have a synchronization pulse at a fixed rate which is not related 
Kicker

AGS ramp

Switch magnet

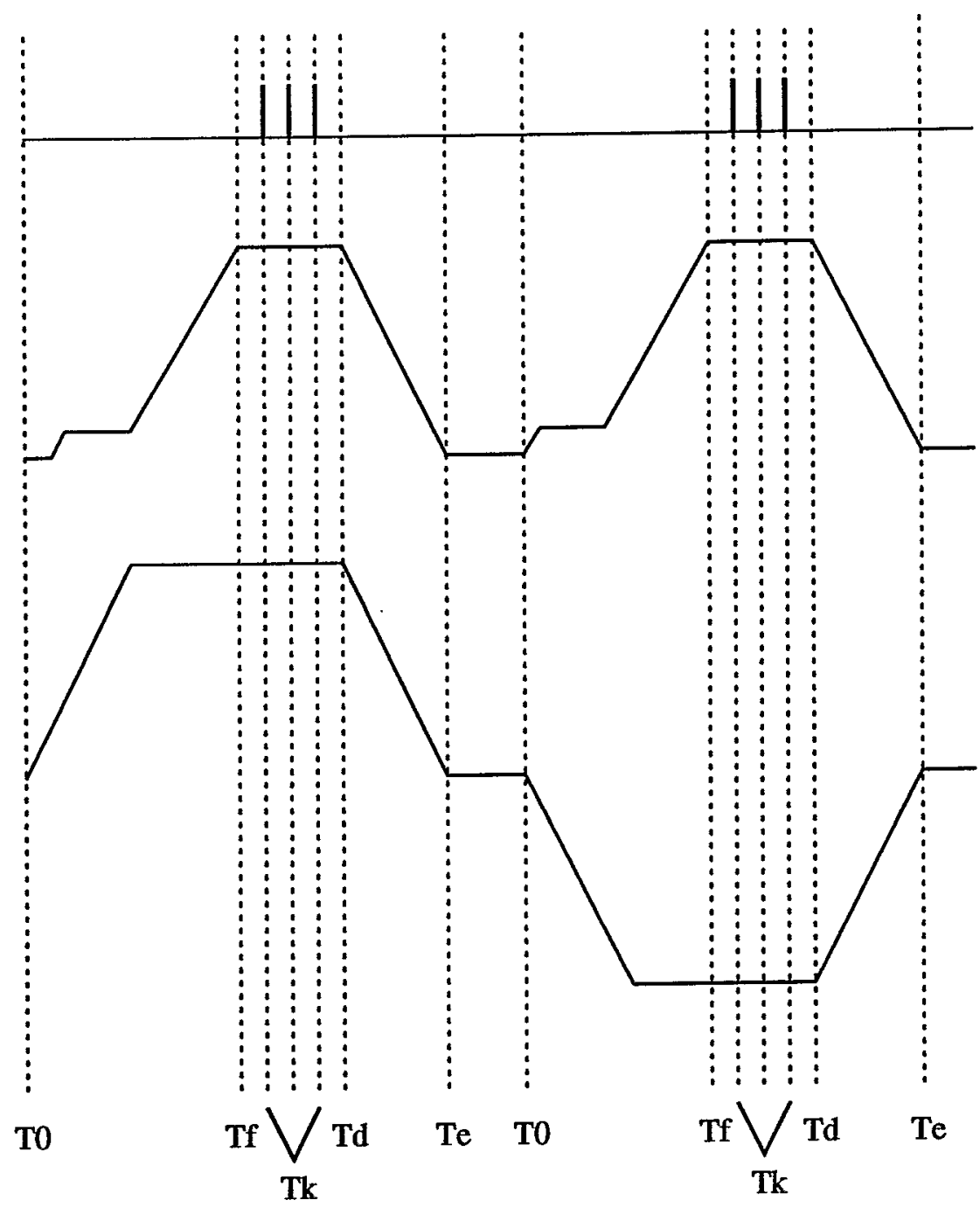

Fig. 1. Schematic timing of the AGS and switching magnet ramps, as well as the kicker pulses. TO and Te correspond respectively to the beginning and end of the AGS cycle. The beginning and end of the flattop are indicated by $\mathbf{T f}$ and $\mathbf{T d}$, respectively. The kicker warning events are indicated by $\mathbf{T k}$.

to the AGS cycle. This would be used to inform the DSP boards to reread any registers which may have been reloaded. Does this really need to be a continuous stream of events, or should it be a software generated event instigated by the program which loaded the registers? Perhaps this type of event is necessary for the RHIC rings, but for the ATR the reading of parameters should be in synch with the TO pulse. Settings should not be changed in the middle of a flattop!

Chris Saltmarsh requested that a provision be made for generating timing events independently from the AGS timing system, so that debugging could take place without interfering with the normal AGS operation. Don Barton seems to think that this is unnecessary, since the system is programmable and software generated events may be used without interfering with the AGS cycle. I must admit that I am a bit skeptical on this point, since some of our problems may be related to how fast we can process and read data 
from the flags. This may require controlling the repetition rate of the $\mathbf{T 0}$ event.

My current thoughts are that as a minimum we need to have the three event types: T0 for the start of a cycle, Tk for the warning of an impending beam pulse, and Td for the beginning of a down ramp and mopping up data. Additionally there will be a few events required to operate the WFG's, but we will spell this out a little better as development progresses. For the ATR the T0 events should provide synch pulses, although a software generated event could also be used for debuggery and later RHIC operation. 